\title{
Quark Matter in Compact Stars
}

\author{
Thomas Klähn* \\ Institute for Theoretical Physics, University of Wroctaw (Poland) \\ E-mail: thomas.klaehn@gmail.com
}

\begin{abstract}
Neutron stars by their very nature are the most likely physical systems in nature where pure, deconfined quark matter might be realized. Over the last years, it became increasingly clear that, despite newly arising observational data it is a challenging task to identify signals which can be interpreted as a clear indicator for or against the existence of this state of matter in neutron star cores. The reasons for this difficulty are manifold and are connected to both, the observational situation as well as the current state of the art regarding neutron star matter equations of state. I give a brief review about constraints on the stiffness of the equation of state of matter in neutron stars and heavy ion collision and discuss possible consequences for the quark matter equation of state. Finally, a very brief outlook questioning the actual impact of presently common mean field approaches is given.
\end{abstract}

Sixth International Conference on Quarks and Nuclear Physics

April 16-20, 2012

Ecole Polytechnique, Palaiseau, Paris

${ }^{*}$ Speaker. 


\section{Introduction}

Neutron stars (NS) are remnants of Supernovae (SN) explosions. The earliest possible SN observation dates back as far as $185 \mathrm{CE}$ when Chinese astronomers recorded the appearance of a bright star, disappearing after a couple of month. A similar event happened in $1054 \mathrm{CE}$ which has been recorded in many places all over the world, - Asia, Middle Asia, America and as I recently learned Europe ${ }^{1}$. These records, mostly written witness, may differ regarding the exact time of the event and the quality of the description, but the mere fact, that numerous of these reports exist illustrates how SN fascinated ancient observers as they still fascinate the 'modern' scientist. The remnant of SN 1054, also known as Crab nebula including the Crab pulsar PSR B0531+21 is a well suited example to point out some of the difficulties compact star studies provide and what an extreme environment a NS is. While we slowly begin to understand the mechanisms which cause SN explosions and lead to the birth of NS great parts of the internal physics of NS are best understood as a number of riddles.

The riddle I am interested in, is the question whether NS might have an inner core consisting of quark matter or other exotica. The answer to this question does not yet exist. But as it turns out, looking for the answer gives interesting insights into topics which one did not originally intend to deal with. In this contribution I will exemplify this by pointing out the potentially extremely fruitful interplay between the research of heavy ion collisions and the properties of neutron stars. Convinced, that this approach is suited to support any future efforts I will try to raise cautious doubts on the reliability of the currently often perceived as state of the art approach of describing dense, cold, deconfined quark matter within effective mean field models.

\section{Dense Matter Phenomenology - Neutron Stars and HIC}

One of the better accessible constraints on the EoS of NS matter is the maximum of observed neutron star masses in binary systems. The reason for this is the direct connection between the stiffness of the EoS and the maximum NS mass one can obtain for this EoS. This is illustrated in Fig. 1 where both, the energy per particle in symmetric matter, as well as the resulting mass configurations for neutron stars are plotted. As can be seen, the stiffness and maximum mass correlate almost perfectly. At this point I want to point out two facts which sometimes are perceived as being confusing. The first thing one notes is, that in Fig.1 the energy per particle of symmetric matter is plotted while the symmetry energy, which gives the energy contribution due to an isospinasymmetry is neglected, even though NS are the most isospin-asymmetric system one can think of in nature. This is simply because this contribution to the EoS at high densities is comparable small. Further, when one speaks about a high necessary stiffness of EoS in order to obtain high NS masses it is not (necessarily) meant, that the compressibility $K$ is large, even though this is helfpful, of course. This statement can rather be understood as a requirement on the slope of the energy per particle at supersaturation densities. However, a clear definition of stiffness in this context does not exist and the term is a rather qualitative judgement.

\footnotetext{
${ }^{1}$ Concentrated information regarding ancient $\mathrm{SN}$ observations is not easily found in physical journals. Wikipedia and google provide good sources for an initial search regarding this topic.
} 

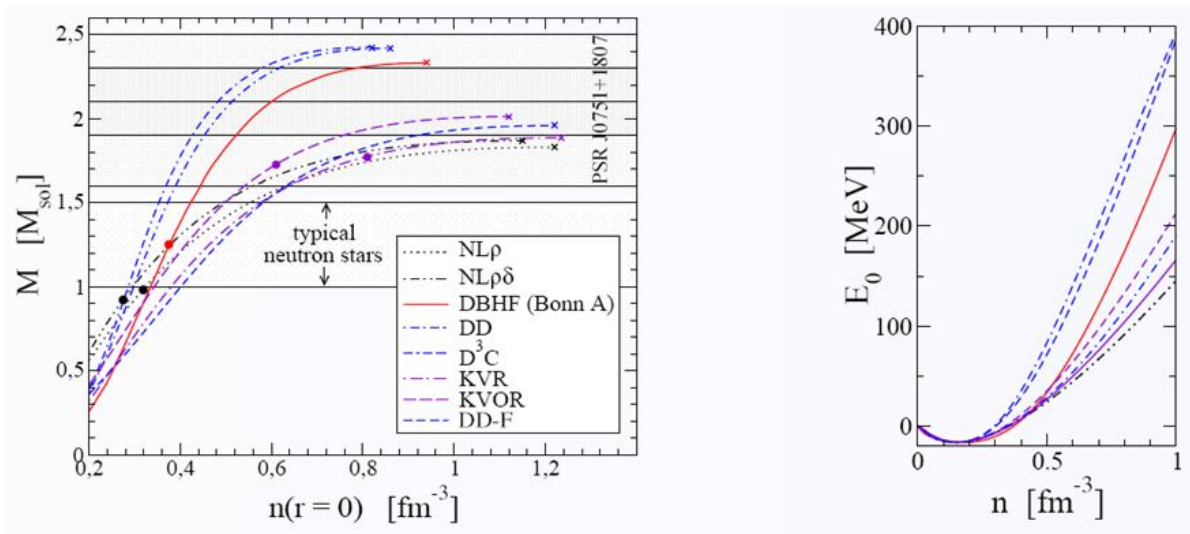

Figure 1: The stiffness of the symmetric matter EoS (right panel) correlates with the maximum neutron star mass (left panel). The symmetry energy has minor impact on $\mathrm{M}_{\max }$. All EoS are referenced in [1].

The maximum mass constraint on the minimum stiffness of the EoS became very severe after a mass of $M=1.97 \pm 0.04 M_{\odot}$ has been reported for PSR J1614-2230 with an unprecedented accuracy in this high mass regime [2] and without perceivable objections from expert groups involved in this field. Shortly after the measurement became public implications have been discussed in [3]. Interestingly, the sometimes heard statement, that EoS of exotic matter, as hybrid EoS accounting for Kaon condensation or any state of quark matter would soften the overall EoS to a degree where it cannot support NS of this or a comparable mass has been clearly contradicted in this work. I'd like to take the opportunity to support this statement once more and to state clearly, that, although in general a phase transition implies a softening of the EoS it is not mandatory that this prevents the existence of massive NS. Moreover, the current understanding of these exotica is by far not advanced enough to draw far reaching conclusions either in favor or disfavour of the existence of exotic matter in NS cores. The best thing, which can be currently done is to analyse model EoS in detail with respect to its available parameters and broadly with respect to the consequences for the currently available phenomenology.

Eventually this has to lead to parameter studies for specific models or even whole classes of models. We performed a study like this for a simple NJL type model [4] following the question for which parameter range of all possible values for the diquark- and vector coupling one could find quark matter cores and, if a quark matter core is present, how massive it would be. The outcome of this study is illustrated in Fig.2 Besides the obvious outcome - strong vector couplings support massive quark matter cores - we found a less obvious and interesting result. The minimal transition density in symmetric matter for configurations which support quark matter cores for massive NS in this specific model is almost independent of the actual value of the vector coupling. In Fig.2 this becomes evident, as one can easily see, that if this density is clearly below four times saturation density, no combination of $\eta_{V}$ and $\eta_{D}$ would result in massive enough NS configurations. I want to stress the point, that this is the transition density in isospin-symmetric matter as investigated in HIC! It appears to be a generally good and promising approach to incorporate all possible known results from HIC studies (and maybe even some unknown, like just shown).

According to this philosophy, a further constraint on the stiffness of the EoS which appears to 


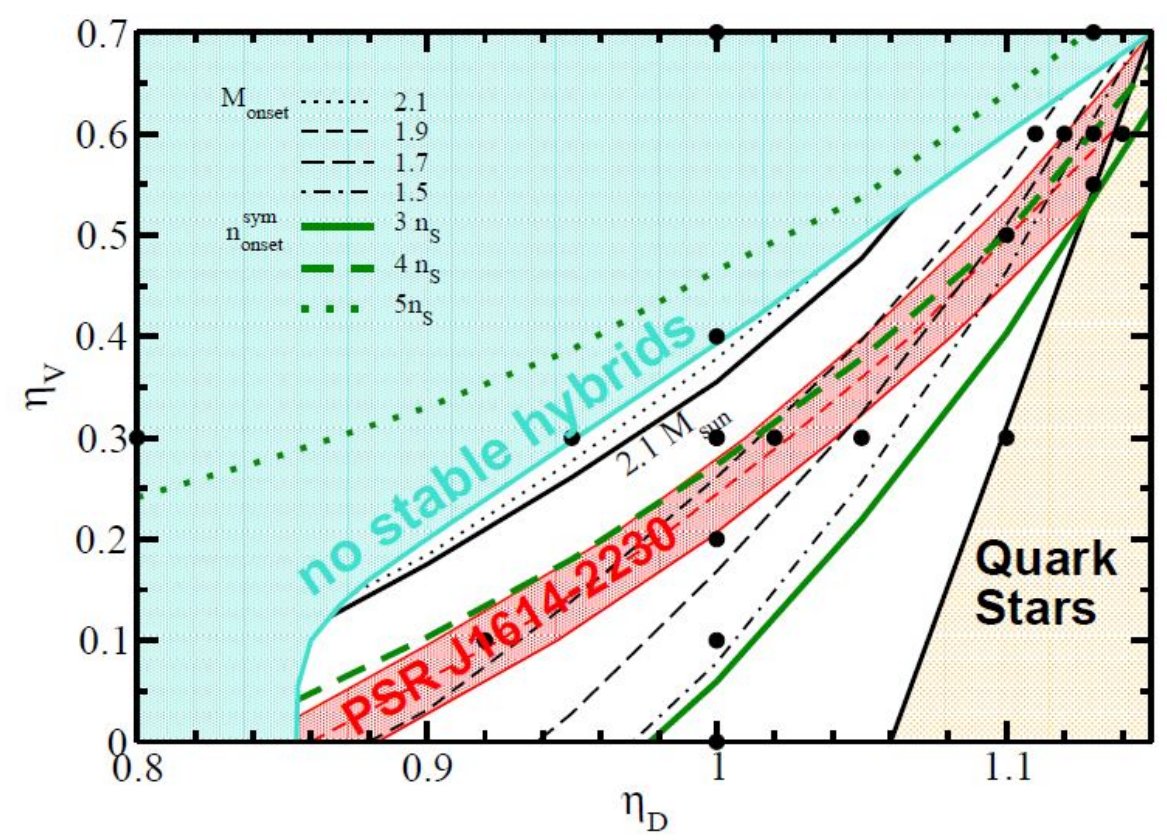

Figure 2: The overall result of the parameter study of the NJL model concerning the vector $\left(\eta_{V}\right)$ and diquark $\left(\eta_{D}\right)$ channel coupling. The red band and all parameter pairs over it correspond to EoS which reproduce at least the $1.97 \pm 0.04 M_{\odot}$ of PSR J1614-2230. No stable hybrid configurations are found in the cyan region, while the grey hatched region corresponds to a very early onset of quark matter. The onset of quark matter in symmetric matter, indicate by green lines suggests, that knowing the actual transition density can give a valuable hint to solve the riddle of the existence of QM in NS. A more detailed discussion is given in [4].

be useful arises from flow data analysis of dense symmetric nuclear matter probed in HICs [5]. It reveals a region in the pressure density diagram of cold matter which is supported by this analysis. This is in particular useful as very stiff EoS tend to result in pressures too high at a given density. Therefore, considering the maximum mass and the flow constraint together one has a useful tool which limits the stiffness of dense cold matter to a remaining, but still great amount of EoS which are neither too stiff (flow) nor too soft (maximum mass). In [6] we eploited the flow constraint in order to parameterize an equation of state first such, that it is in agreement with the flow constraint and verified after this, that it fulfills a two solar mass NS constraint as well. The result has been satisfying, consequently both conditions have been met as can be seen in Fig.3.

\section{Quark Matter - Unconstrained}

So far I have presented only results which rather describe a strategy how to exploit two useful constraints on the equation of state arising from massive NS observations and the analysis of flow data. This approach is useful in general and for every likely and unlikely EoS of dense cold matter. The important result is, that quark matter (as probably most other exotica) in NS at our current point of understanding is a possibility which can not be easily rejected. Neither can it be concluded. The truth is, that we don't know and the answer to this question depends on the model we use and the parameterization we choose. Further constraints, most likely concerning transport properties 

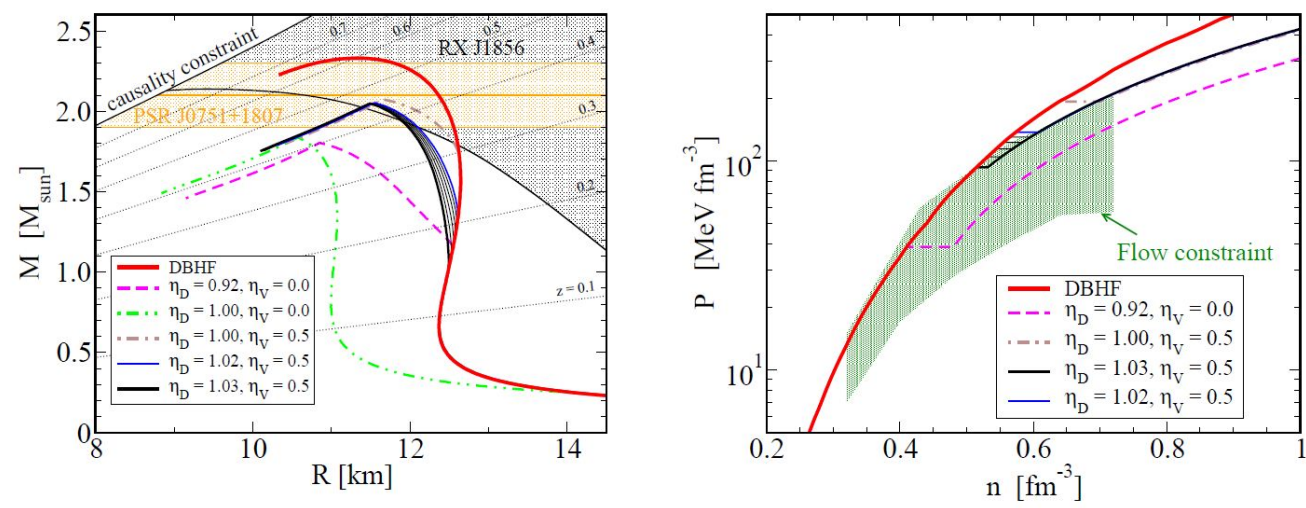

Figure 3: A hybrid EoS parameterized to fulfill the flow constraint (right panel) and a two solar mass NS constraint (warning: at this time PSR J0751+1807, a meanwhile outdated result!). Details in [6].

and therefore the actual composition of a NS which is not very relevant for maximum masses, are very likely to be necessarily considered before one can find a satisfying answer to this riddle. However, transport properties are essentially microscopic quantities and should consequently be calculated within an adequate theoretical framework. When it comes to quark matter degrees of freedom it is clear, that QCD is the proper theory. However, the problems of solving this theory are manyfold, often discussed and in particular at zero temperature and finite densities best described as 'demoralizing'. It is therefore plausible, that in order to make any progress on this field, effective approaches are applied. Concerning quark matter, models of the Nambu-Jona-Lasinio (NJL) type have been widely investigated. Their great advantage is their ability to describe the breaking of chiral symmetry with decreasing density, one of the certainly expected properties of quark matter. The initially missing other key feature of QCD, a transition from confined to deconfined matter at a certain point is mimicked by applying extensions of the NJL model with the zero density Polyakovloop (resulting in the PNJL model). Being a convinced applicant of these effective approaches myself, I see long terms problems arising if one tends to take any of the obtained results as being more than just model studies. In the end of this contribution I want to give one example for these concerns. NJL type models as they are currently applied are mean field models, which means that in particular any gap (like the scalar mass gap which gives access to the effective quark mass) is inherently momentum independent. Effectively, this results in equations of state which differ from a free Fermi gas essentialy only in mean field shifts. In particular, one will always and only find one particle distribution functions with the classic 'Fermi-edge behaviour' a zero temperature, where the edge is defined by the chemical potential. In [7] and [8] we applied different kind of models, both obtained within the Dyson-Schwinger approach in a certain set of simplifications. Dyson-Schwinger equations are derived directly from the QCD-Lagrangian (for details, see both publications and references therein). Even though following approximations have to be made, they prove successful in describing properties of quark bound states, give access to certain criteria connected to the deconfinement transition and, and this is the point I want to stress, have momentum depend gap solutions which directly affect the shape of the one particle distribution function. This is illustrated in Fig.4 where one clearly sees that none of the cases resembles the typical behaviour of an ideal Fermi gas at zero temperature. As this function is crucial for many transport properties, 

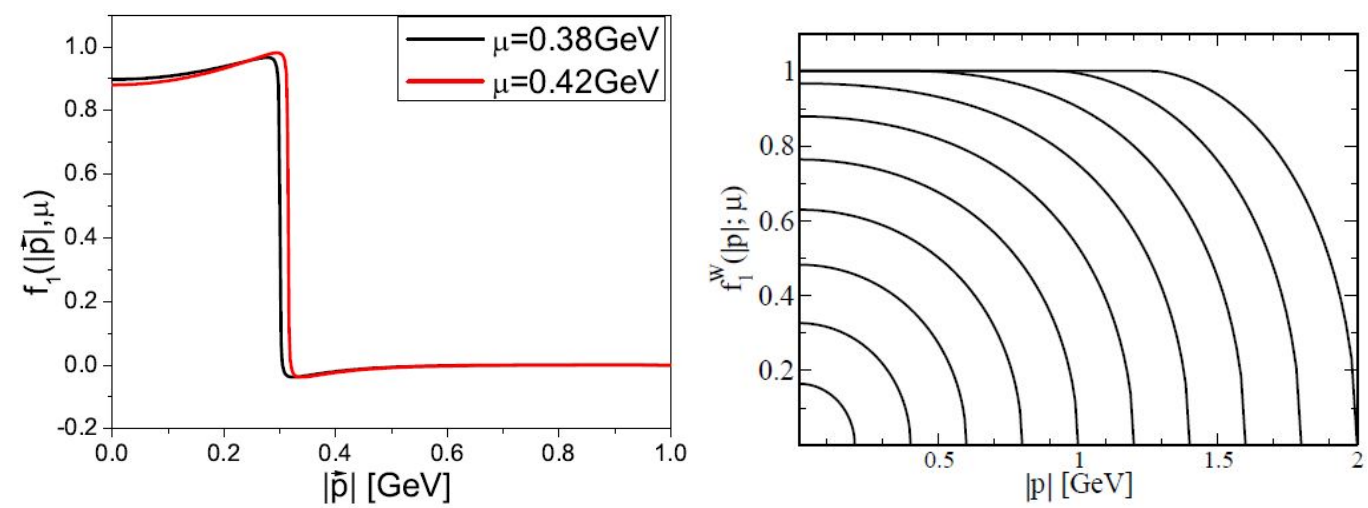

Figure 4: One particle distribution function $f_{1}$ obtained within the Dyson-Schwinger approach for different models ([7] left, [8] right) for various chemical potentials. Both base on different approximations but none of them shows the 'classical' Fermi-edge at the chemical potential.

the consequences might be severe.

\section{Acknowledgement}

The author is grateful to all his collaborators of the here cited publications and is supported by the network "hadronphysics3" within the seventh framework programme of the European Union.

\section{References}

[1] T. Klahn, D. Blaschke, S. Typel, E. N. E. van Dalen, A. Faessler, C. Fuchs, T. Gaitanos and H. Grigorian et al., Phys. Rev. C 74, 035802 (2006) [nucl-th/0602038].

[2] P. Demorest, T. Pennucci, S. Ransom, M. Roberts and J. Hessels, Nature 467 (2010) 1081 [arXiv:1010.5788 [astro-ph.HE]].

[3] J. M. Lattimer and M. Prakash, arXiv:1012.3208 [astro-ph.SR].

[4] T. Klahn, D. Blaschke and R. Lastowiecki, arXiv:1111.6889 [nucl-th].

[5] P. Danielewicz, R. Lacey and W. G. Lynch, Science 298, 1592 (2002) [nucl-th/0208016].

[6] T. Klahn, D. Blaschke, F. Sandin, C. Fuchs, A. Faessler, H. Grigorian, G. Ropke and J. Trumper, Phys. Lett. B 654, 170 (2007) [nucl-th/0609067].

[7] H. Chen, W. Yuan, L. Chang, Y. -X. Liu, T. Klahn and C. D. Roberts, Phys. Rev. D 78, 116015 (2008) [arXiv:0807.2755 [nucl-th]].

[8] T. Klahn, C. D. Roberts, L. Chang, H. Chen and Y. -X. Liu, Phys. Rev. C 82, 035801 (2010) [arXiv:0911.0654 [nucl-th]]. 